\title{
DICUSSION C. The stellar yields in He-3, He-4 and Li-7: main sources, observational constraints, and problems
}

\author{
André Maeder \\ Geneva Observatory, University of Geneva, CH-1290 Versoix, Switzerland \\ email: andre.maeder@unige.ch
}

Keywords. Nucleosynthesis, abundances

I am pleased to recall that the first determination of the $\mathrm{Li}$-abundance in the Sun was made at the Geneva Observatory in 1975 by Edith Müller, Eric Peytremann and Ramiro de la Reza on the basis of spectra taken at Kitt Peak. The first Be determination was also made in Geneva the same year by Y. Chmielewski, J. Brault (Kitt Peak National Observatory) and E. Müller. These two outstanding works opened the door for all further investigations on these light elements.

The yields of light elements are a matter where the shock of ideas frequently occurs, with new facts arising and different interpretations. This is particularly the case at present concerning the He abundance in globular clusters and the relation between the ${ }^{7} \mathrm{Li}$ abundances and planets. This made the discussions at IAU Symposium very lively and interesting. I will try my best to summarize the main points, restore the essence of the discussions and give a proper credit to the various contributors.

Normally, stellar evolution provides the chemical yields which are used as an input for the models of the chemical evolution of galaxies. The inverse path, i.e. using the observations of galactic trends in chemistry to infer the stellar properties is like trying to derive the properties of an egg from an omelette, as stated by Jean Audouze long time ago. Nevertheless, in the case of the very early stellar generations, the only direct infos we often have on the stellar properties come from the observed trends in the chemical evolution of galaxies. In this case, we can even say that we use a "fossil omelette" to reconstruct the original stellar properties of the very early stellar generations.

\section{The yields in He-4, the globular clusters, and related questions}

The relative helium to oxygen enrichment $\Delta Y / \Delta O$ obtained by Manuel Peimbert is $3.3 \pm 0.7$, a ratio which typically applies to stars with solar abundances. We notice that the yields in $\mathrm{He}$ and $\mathrm{O}$ do not come from the same mass range, and that the above ratio depends heavily on mass loss, mixing, mass limit for black hole formation, cutoff mass in supernovae, etc... The question of the slope of the $\Delta Y / \Delta Z$ according to the metallicity $Z$ is raised by Francesca Matteucci. Manuel Peimbert points out that $\Delta Y / \Delta Z$ may reach a value of about 4 at $Z$ higher than solar.

The big question concerns the multiple sequences found in globular clusters, following the VLT study of $\omega$ Cen by Piotto and colleagues. The globular clusters have experienced successive starburst episodes, the recent one bearing the signature of strong He-enrichments. The red sequence corresponds to $Z=10^{-3}, Y=0.246$, while the blue sequence has $Z=2 \cdot 10^{-3}, Y=0.38$. Formally, this corresponds to a large He-enrichment for a minute one in heavy elements, i.e. $\Delta Y / \Delta Z \sim 10^{2}$. The discussions bear, not on the reality of the high He-content, but rather on its origin. Some authors in literature support the idea that the galactic winds driven by supernovae have removed the contributions in heavy elements, because they are ejected at high speed. Manuel Peimbert notes the $\mathrm{C} / \mathrm{O}$ ratios in the gas may provide information on the importance of 
the ejecta of heavy elements in the galactic winds. The observations show low $\mathrm{C} / \mathrm{O}$ ratios, which indicates that there is no important losses of $\mathrm{O}-$ rich material by the galactic winds. The material forming the ejected galactic winds is likely rather well mixed. Francesca Matteucci notes that the same remark seems to also apply to dwarf galaxies. Donatella Romano nevertheless mentions there are observations in a dwarf galaxy of galactic winds showing typical SN II ejecta.

One may wonder whether the galactic infall could not counterbalance the effects of selective ejecta. Monica Tosi emphasizes that all galaxies experience infalls, while only a few show galactic winds. She points out that dwarfs galaxies tend to develop galactic winds. She mentions that the above mentioned $\mathrm{C}$ observations are made in nearby galaxies, where there is likely no galactic winds.

Georges Meynet supports a different idea, i.e. that fast rotating massive stars at low $Z$ lose a lot of mass as a result of rotational mixing and mass loss, thus making the He yields very large. He also relates these excesses to the anomalies of the CEMP (Carbon rich extremely metal poor stars). Some authors support losses from AGB stars or contributions from binaries. He emphasizes that massive and AGB stars do not produce exactly the same chemical anomalies and that this may be a way to disentangle the two types of contributions.

Why do these large He abundances do not affect the general $\Delta Y / \Delta Z$ ratio? Is it because the fraction of stars which form under the above peculiar conditions is too small? This seems likely, however this could be the source of some scatter in the $Y$ vs. $Z$ relation at low $Z$. Francesca Matteucci remarks that the peculiar solutions for the globular clusters do not necessarily apply to the Galaxy and she emphasizes the interest to perform some tests in the Galactic bulge.

Among other points, Marc Pinsonneault emphasizes the interest to get He abundances from asteroseismology in all possible objects. Nikos Prantzos remarks that the Sun places very good constraint on the $\Delta Y / \Delta Z$ ratio. This gives a value within the error bars of that given by Manuel Peimbert.

\section{The surprising flat behaviors of the $\mathrm{He}-3 / \mathrm{He}$ ratio}

In solar-type stars, a bump of ${ }^{3} \mathrm{He}$ forms at mass fraction 0.55 and the standard models predict that this ${ }^{3} \mathrm{He}$ should be ejected in the more advanced stages. The massive stars on the contrary destroy ${ }^{3} \mathrm{He}$ over most of their interior, both the initial cosmological one and the new amounts they may have synthesized, thus their yields are negative. The net result of the standard models integrated over the mass spectrum, as shown by models of the chemical evolution of galaxies made by Monica Tosi, is that the ${ }^{3} \mathrm{He} / \mathrm{He}$ ratio should increase with the $[\mathrm{O} / \mathrm{H}]$ abundance ratio. In the Galaxy, this ratio should decrease with the galactocentric distance.

However, the problem is that more than $90 \%$ of the solar-type stars show no ${ }^{3} \mathrm{He}$ enrichement or at least much less than predicted. Among the planetary nebulae, only a few ( 7 as discussed by Tom Bania) show ${ }^{3} \mathrm{He}$ enhancements in agreement with the current expectations. Also contrary to the model expectations, the ${ }^{3} \mathrm{He} / \mathrm{He}$ ratio is flat with respect to $[\mathrm{O} / \mathrm{H}]$ as discussed by Gary Steigmann and Tom Bania. The ${ }^{3} \mathrm{He} / \mathrm{He}$ ratio is similar to that given by the standard Big Bang nucleosynthesis. Also, this ratio does not show the expected variations with the galactocentric radius or with the oxygen abundance. This is the so-called He-3 problem, the reality of which is not disputed.

Gary Steigman emphasizes that ${ }^{3} \mathrm{He}$ is both produced and destroyed, but in a way that the net production is zero, so that it keeps to the level of the standard Big-Bang synthesis. Corinne Charbonnel supports these views. She emphasizes that the low-mass stars have small positive yields (even with the thermohaline mixing), thus they do not destroy the cosmological ${ }^{3} \mathrm{He}$. The planetary nebulae with the predicted ${ }^{3} \mathrm{He}$ abundances also have the classically predicted ${ }^{12} \mathrm{C} /{ }^{13} \mathrm{C}$ isotopic ratios. In relation with her models, Corinne Charbonnel mentions that it would be interesting to know the magnetic fields in RGB stars with the classical ${ }^{12} \mathrm{C} /{ }^{13} \mathrm{C}$ isotopic ratios.

Monica Tosi mentions that the present HII regions exhibit no ${ }^{3} \mathrm{He} / \mathrm{He}$ gradient in the Milky Way, she emphasizes this confirms that there is no net overall production of ${ }^{3} \mathrm{He}$ at the present epoch. Manuel Peimbert questions whether there are biases in the studies of the planetary 
nebulae showing nitrogen enrichments. Tom Bania confirms that the sample of observed planetary nebulae is currently biased in favor of those showing nitrogen enrichments.

A possible solution for the ${ }^{3} \mathrm{He}$ problem is that proposed by Nadège Lagarde, Corinne Charbonnel, and Jean-Paul Zahn, it implies an instability due to thermohaline mixing. This instability, like salt fingers, occurs when matter with an higher mean molecular weight $\mu$ lies above matter with a lower $\mu$. The reaction ${ }^{3} \mathrm{He}+{ }^{3} \mathrm{He} \rightarrow{ }^{4} \mathrm{He}+2 \mathrm{p}$ results in the conversion of two particles into three ones at the basis of the ${ }^{3}$ He bump. Thus, there it reduces the $\mu$-value making a local inversion which creates an instability. One may note that this solution is reminiscent of the so-called "solar spoon" proposed decades ago by D. Gough.

However, the question arises why do some planetary nebulae still have the expected ${ }^{3} \mathrm{He}$ enrichments. The answer by Corinne Charbonnel is that this may be due to a magnetic field inhibiting the thermohaline instability. She proposes that these planetary nebulae are the descendants of the magnetic Ap stars. In both cases, the proportions of these objects are about $5 \%$. Further investigations are needed to test these possibilities.

\section{Questions regarding lithium: the Li plateau, Li in RGB and AGB stars, relation with planets}

It is remarkable that thanks to the efforts of many people we now seem to have a consistent interpretation of the various physical effects shaping the $\mathrm{Li}$ abundance in the $T_{\text {eff }}$ region surrounding the Li dip (see Fig. 1).

When one refers to the (in principle) unmodified $\mathrm{Li}$ abundance, it means the abundance on the left side of the Li dip as shown in the Figure. These Li abundances for stars covering a large range of $[\mathrm{Fe} / \mathrm{H}]$ show an essentially flat distribution over the whole range of metallicities except their increase for the higher $[\mathrm{Fe} / \mathrm{H}]$ corresponding to Pop. I stars. The almost flat part is the so-called "Spite plateau". The big problem is that this abundance plateau lies a factor of about 3 below the predictions of standard Big-Bang nucleosynthesis. In this respect, there is an interesting poster by Sbordone et al. on the low-metallicity end of the Spite plateau. Below $[\mathrm{Fe} / \mathrm{H}]=-3$, he notes that there is no plateau anymore, but a growth of $\mathrm{A}(\mathrm{Li})$ with $[\mathrm{Fe} / \mathrm{H}]$ and a large scatter.

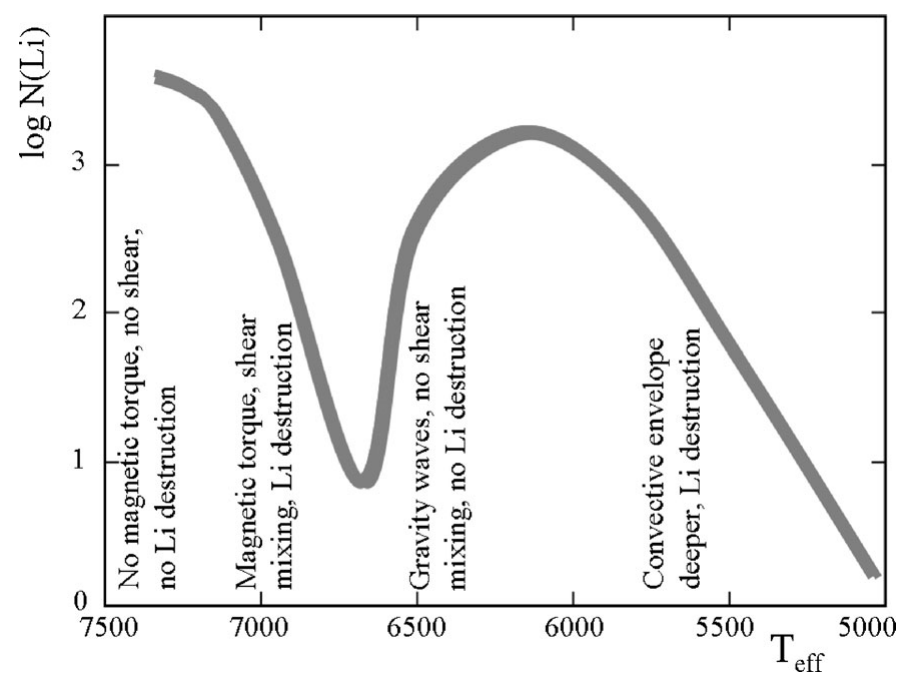

Figure 1. The physical effects shaping the Li dip as a function of the effective temperature. Adapted from S. Talon et C. Charbonnel. 
The main lesson concerning the $\mathrm{Li}$ abundance $A(\mathrm{Li})$ on the hot side of the dip is that it appears to be a multivariate function of the form

$$
A(\mathrm{Li})=f(\text { mass }, Z \text {, age, rotation, pre }-M S, \text { disk lifetime }, \ldots),
$$

some effects being still disputed. The evolutionary effects along the tracks in the HR diagram are clearly predicted by the stellar models and they are also observed along the sequences of globular clusters. The $T_{\text {eff }}$ scale is of particular importance along the subgiant branch, as emphasized in several interventions in the discussion.

There are clear differences of views on the age effect on the Li-abundances. For Sofia Randich, each cluster behaves in a different way and the relation with age is uncertain. Jorge Melendez however shows that the $\mathrm{Li}$ abundances for the available solar twins of the Hipparcos catalogue present a strong correlation with age in agreement with the Charbonnel \& Talon (2005, Science, 309, 2189) models.

Andreas Korn wonders whether the Li dip is also observed in PopII stars as in Pop I stars. Suzanne Talon answers that Pop II stars are so old that the Pop II stars with masses at the level of the Li dip already occupy the subgiant branch, thus it may not be easy to see the it. Corinne Charbonnel further comments that the dip has nevertheless been found in halo stars (Charbonnel \& Primas, 2005, A\&A, 442, 961) and in NGC 6397 by Lind et al. (2009, A\&A, 503, L545).

There are theoretical and observational evidences of some additional lithium depletion near the bump of the red giant branch (RGB) and various mechanisms are proposed to account for this depletion, in particular the hot-bottom burning and the thermohaline mixing. There is a variety of $\mathrm{Li}$ abundances in RGB and AGB stars. $1 \%$ of the GK giants are Li-rich, there are also Li rich AGB stars, which may be related to the Cameron-Fowler mechanism. To a question concerning the origin of the Li-depleted stars, Marc Pinsonneault suggests they are mergers.

Francesca Matteucci then makes some comments regarding $\omega$ Cen and the related problems. One may recall here that in his presentation Piercarlo Bonifacio has suggested, from the similarity of the Li content in $\omega$ Cen and in the Galactic halo field stars (which have experienced different evolutionary histories), that there is no evidence of a pre-galactic Li depletion by a generation of very massive stars as was suggested by Laurent Piau. Bonaficio has also noted the absence of difference in the Li content of stars in $\omega$ Cen over a range of 5 Gyr in ages, he has claimed that this in contradiction with the current explanations of the Li plateau based on diffusion processes. Francesca Matteucci wonders about the assumed 5 Gyr scatter and also on the sampling of data.

The last topic approached in this discussion concerns the relation between the enhanced Lidepletion and the occurrence of planets in solar-type stars. This interesting result was shown by Garik Israelian and also presented by Nuno Santos in his review. The point is however challenged in a poster by Jorge Melendez.

There are several possible interpretations of the above fact. The merging of a giant planet may bring additional angular momentum in the outer layers, thus driving mixing and Li-depletion, also the pollution resulting from the merging may give rise to thermohaline mixing as was suggested by Sylvie Theado and Sylvie Vauclair. Marc Pinsonneault makes comments on the importance of the disk and of rotation in the pre-Main Sequence evolution. He emphasizes the need to measure rotation rates. The key parameter (as firstly emphasized by Jerome Bouvier) is the disk lifetime. A longer disk lifetime means more disk locking in the pre-MS phase and thus a lower mean rotation on the ZAMS. The longer disk lifetime also leads to higher mixing due to more differential rotation in slowly rotating stars. Another consequence of the longer disk lifetime is that there is more possibilities for the formation of planets. This may establish a relation between the Li-depletion and the occurrence of planets via the longer disk lifetime.

This discussion was a beautiful illustration of the major open questions remaining in our understanding of the abundances of the light elements. I thank Patrick Eggenberger for his help in taking notes of the session. I apologize for any deviation from the exact meaning of the contributors, I hope nobody will bring me to the court for that. 